\title{
Análisis de la deformabilidad del acero inoxidable AISI 304 DDQ en condiciones multiaxiales de embutición. Evaluación de la influencia de la acritud inicial ${ }^{(\bullet)}$
}

\author{
J. Coello*, V. Miguel*, A. Calatayud*, A. Martínez* y C. Ferrer**
}

Resumen

\begin{abstract}
La embutibilidad de un material se establece en función de indicadores normalizados, como son los índices de anisotropía y de acritud, que suelen determinarse en condiciones diferentes a las de conformado. Por otro lado, también es muy frecuente determinar la embutibilidad de los materiales mediante ensayos simulativos, que no separan las diferentes variables que actúan en el proceso como, por ejemplo, la dirección de laminación. En el presente trabajo se utiliza un ensayo con matriz en forma de cuña para determinar el coeficiente de endurecimiento y de anisotropía en función de la dirección de laminación para diferentes deformaciones en condiciones similares a las de tracción-compresión biaxial existentes en la zona del ala de un proceso de embutición profunda. El material estudiado es acero inoxidable AISI 304 con calidad de embutición. También se establece la influencia existente para estados de acritud inicial diferentes, laminación y tracción biaxial. Los resultados obtenidos permiten establecer la validez del ensayo realizado desde el punto de vista de la deformación que impone al material. También, se demuestra el efecto predominante de la acritud inicial del material frente al provocado en condiciones multiaxiales de embutición y se establece la variación del coeficiente de anisotropía del material con la deformación de embutición para la dirección coincidente con la de laminación del material.
\end{abstract}

\section{Deformability analysis of the AISI 304 DDQ stainless steel under deep drawing multiaxial condition. Evaluation of the initial strain influence}

\begin{abstract}
The deep drawing formability of a material is established as a function of standard indexes, as strength coefficient and anisotropy coefficient. But these indexes are determined in different conditions to those that take place in the forming process. The simulative assays do not separate the actions due to the different variables that work in the process, as for example, the rolling direction. In the present work a test that uses a wedge shape die is considered in order to obtain the strength and anisotropy coefficients as a function of rolling direction. This way, the assays are carried out under a tensile-biaxial compression stress state similar to that one taking place in the flange zone in deep drawing. The experimented material is a deep drawing quality stainless steel AISI 304. The influence of initial strengthened states, rolling and uniaxial tensile on the steel behaviour are also studied. The results permits the authors establish the validity of the assay from the point of view of the strains produced in the sheet. The initial strain has a higher effect on the material than that one obtained from the tensile-biaxial of the state than the tensile-biaxial compression causes. The anisotropy coefficient changes with the strain for the sheet rolling direction.
\end{abstract}

Keywords

Formability; Anisotropy; Initial strain state; 304 DDQ.

\section{INTRODUCCIÓN}

La embutibilidad de un material, generalmente, se establece en función de indicadores intrínsecos a éste, como son el índice de endurecimiento de Hollomon, n, y de anisotropía o de Lankford, r. Chiang y
Kobayashi $^{[1]}$ establecen que la relación límite de embutición aumenta con r y, en menor medida, con n. Woo ${ }^{[2]}$, afirma que un elevado coeficiente de endurecimiento representa un comportamiento del material, potencialmente adecuado para procesos de conformado en condiciones de tracción biaxial,

(•) Trabajo recibido el día 15 de Diciembre de 2009 y aceptado en su forma final del día 16 de Abril de 2010.

* Instituto de Desarrollo Regional; Universidad de Castilla-La Mancha; Avda. España s/n 02006 Albacete.

**Departamento de Ingeniería Mecánica y de Materiales; Universidad Politécnica de Valencia; Camino de Vera 46022 s/n. 
permitiendo una distribución más uniforme de las deformaciones. Los indicadores de embutibilidad, r y n, se emplean como datos de partida en la modelización del proceso de embutición mediante métodos numéricos $^{[3}$ y 4$]$. Sin embargo, para la obtención de estos parámetros, generalmente, el material es sometido, únicamente, a esfuerzos de tracción uniaxial ${ }^{[5]}$, que representa condiciones muy diferentes de las que se dan en el proceso de embutición. Como consecuencia de ello, la información que aportan es limitada.

J-L. Geoffroy et al. evalúan la conformabilidad de los materiales a partir de las curvas límite de conformado, FLC, basadas en la simulación mediante elementos finitos. No obstante, las condiciones en las que generalmente se obtienen, presencia de deformaciones lineales, no existencia de fricción, deformación homogénea en espesor no siempre se cumplen en los procesos de embutición, por lo que deben ser consideradas con cautela en la predicción de comportamientos en el conformado ${ }^{[6]}$. Aunque algunos trabajos persiguen condiciones representativas del proceso de embutición, como es la deformación plana, dichas condiciones no se reproducen exactamente ${ }^{[5]}$. Otro de los problemas fundamentales en relación a la modelización de los procesos de estampación radica en el hecho de que se sigue asumiendo el comportamiento a tracción uniaxial del material como punto de partida para el desarrollo posterior de la teoría de la plasticidad. Existen autores que cuestionan este hecho por la falta de concordancia entre resultados experimentales y numéricos ${ }^{[7 \mathrm{y} 8]}$. Kessler, L. et al. ${ }^{[7]}$ demuestran que existen importantes diferencias entre los resultados experimentales y numéricos obtenidos en el proceso de embutición, justificando estas diferencias por las importantes variaciones existentes entre la respuesta de un material sometido a esfuerzos de tracción uniaxial y esfuerzos típicos de embutición. En este sentido, V. Miguel y et al ${ }^{[8]}$, determinan, experimentalmente, las características mecánicas del acero DC-05 y AISI 304 en condiciones de tracción- compresión biaxial, representativas del proceso de embutición y realiza un estudio de embutición mediante el método numérico de las líneas de deslizamiento, demostrando que en este caso los resultados experimentales son del mismo orden que los numéricos.

También, existen ensayos simulativos que reproducen las condiciones de los diferentes procesos de estampación como estirado biaxial, ensayo Fukui, ensayos de embutición de copas ${ }^{[9]}$, que se emplean para el estudio de variables del proceso por algunos autores pero, estos ensayos no separan bien el efecto de las diferentes variables que intervienen en el proceso. Así, Rajagopal ${ }^{[10]}$ recurre a funciones analíticas para distribuir la carga en el punzón y Gunnarson et al. ${ }^{[11]}$ no aíslan, totalmente, el efecto de la fricción del prensachapas. Aunque numerosos investigadores hacen uso de estos ensayos, en la práctica, solo se utilizan ventajosamente para evaluar lubricantes o para establecer las condiciones que conducen a defectos en el producto acabado ${ }^{[12]}$.

En este trabajo, para estudiar la embutibilidad del acero inoxidable austenítico AISI 304 DDQ, con posibilidad de seleccionar la influencia de las diversas variables que intervienen en los procesos de conformado, se ha establecido una metodología aplicada previamente a acero DC-05 de bajo contenido en carbono, considerando la zona del flanco, aisladamente $^{[13]}$, y la zona de doblado ${ }^{[14]}$. Con objeto de simular la deformación producida en un elemento del flanco de la copa, se han llevado a cabo ensayos de deformación en una matriz de cuña, con ángulos de $15^{\circ}, 10^{\circ}$ y $5^{\circ}$, en la que el material es sometido a tensiones de tracción-compresión biaxial. Mediante esta metodología se han obtenido los índices de endurecimiento y de anisotropía del acero, estableciéndose la influencia de los estados de acritud iniciales del material en el proceso de conformado de la chapa y evaluando la validez de la metodología empleada. Se ha evaluado la modificación de dichos indicadores con la deformación para condiciones de tracción-compresión biaxial, típicas de la zona del flanco del proceso de embutición profunda.

\section{DESARROLLO EXPERIMENTAL}

\subsection{Materiales utilizados}

El material utilizado para el desarrollo de este trabajo ha sido chapa de acero inoxidable austenítico AISI 304 DDQ de espesor, t, 0,8 mm, laminada en frío y con un acabado superficial comercial 2B. Dicho acabado se ha obtenido mediante un proceso de recocido y decapado tras la laminación de la chapa ${ }^{[15]}$. La composición química y las propiedades mecánicas del acero se indican en las tablas I y II. El análisis de composición química se ha determinado mediante espectrofotometría de emisión por plasma en un ICP, Optima 5300 DV. Los ensayos mecánicos se han realizado en una máquina de tracción de $10 \mathrm{KN}$.

Para estudiar el efecto del estado inicial del material sobre el proceso de embutición, el acero AISI 304 DDQ, suministrado por el fabricante, ha sido sometido a un proceso de acritud previa mediante dos procesos de deformación diferentes, laminación y tracción uniaxial. La deformación equivalente de Von Mises, $\varepsilon_{\text {eqo }}$, producida mediante estos procesos, ha sido de 0,090 y 0,205 mediante tracción y de 0,065 y 0,180 por laminación (Tabla III). 
ANÁLISIS DE LA DEFORMABILIDAD DEL ACERO INOXIDABLE AISI 304 DDQ EN CONDICIONES MULTIAXIALES DE EMBUTICIÓN. EVALUACIÓN DE LA INFLUENCIA DE LA ACRITUD INICIAL DEFORMABILITY ANALYSIS OF THE AISI 304 DDQ STAINLESS STEEL UNDER DEEP DRAWING MULTIAXIAL CONDITION. EVALUATION OF THE INITIAL STRAIN INFLUENCE

Tabla I. Composición química (\%) del acero AISI 304 DDQ

Table I. Chemical composition of AISI 304 DDQ steel (\%)

\begin{tabular}{ccccccccc}
\hline $\mathbf{C}$ & $\mathbf{S}$ & $\mathrm{Si}$ & $\mathrm{Mn}$ & $\mathrm{Cr}$ & $\mathrm{Ni}$ & $\mathrm{Mo}$ & $\mathbf{V}$ & $\mathbf{C u}$ \\
\hline 0,040 & 0,003 & 0,19 & 1,11 & 17,67 & 9,01 & 0,28 & 0,08 & 0,26 \\
\hline
\end{tabular}

Tabla II. Propiedades mecánicas e índices de deformabilidad del acero AISI 304 DDQ

Tabla II. Mechanical properties and anisotropy and strengthening coefficients of AISI 304 $D D Q$ steel ( $r$ and $n$ are normal, respectively)

\begin{tabular}{cccccc}
\hline Acero & $\begin{array}{l}\mathbf{R}_{\mathrm{p} 0,2}{ }^{(1)} \\
(\mathrm{MPa})\end{array}$ & $\begin{array}{c}\mathrm{Rm}^{(2)} \\
(\mathrm{MPa})\end{array}$ & HV 0,5 & $\mathbf{r}$ & $\mathbf{n}$ \\
\hline $304 \mathrm{DDQ}(2 \mathrm{~B})$ & 252 & 582 & 174 & 0,906 & 0,244 \\
\hline
\end{tabular}

(1) Límite elástico proporcional al 0,2\%

(2) Resistencia a la tracción.

Tabla III. Deformación equivalente provocada mediante laminación y tracción uniaxial

Table III. Equivalent strain for different deformation systems, rolling and tensile

\begin{tabular}{ccc}
\hline $\begin{array}{c}\text { Proceso de } \\
\text { acritud }\end{array}$ & \multicolumn{2}{c}{$\begin{array}{c}\text { Deformación } \\
\text { equivalente }\left(\mathbf{e}_{\text {eqo }}\right)\end{array}$} \\
\hline Tracción uniaxial & 0,090 & 0,205 \\
Laminación & 0,065 & 0,180 \\
\hline
\end{tabular}

\subsection{Proceso de deformación bajo condiciones de tracción-compresión biaxial}

Con objeto de simular las condiciones de deformación que tienen lugar en un elemento del flanco durante el proceso de embutición profunda de chapa (Fig.1 a)) se ha realizado un ensayo de deformación en condiciones de tracción-compresión biaxial. El ensayo ha sido definido y validado como representativo del proceso para un acero DC-05, bajo en carbono, con calidad de embutición ${ }^{[13 \text { y } 14]}$. Este ensayo está basado en el estirado de probetas planas empleando una matriz en forma de cuña. Mediante un sistema de cierre, se aplica una fuerza normal al conjun- to matriz-pisador, entre los que se aloja la muestra de ensayo. Con la aplicación de una tensión de estirado se hace pasar el material por la matriz, experimentando una deformación plana debido a la compresión lateral de la chapa sin cambio de espesor (Fig. 1 b)).

Se han utilizado matrices con tres ángulos de conicidad, $15^{\circ}, 10^{\circ}$, y $5^{\circ}$ (Fig. $\left.1 \mathrm{c}\right)$ ) Estos ángulos se han establecido en base a la equivalencia geométrica de un sector de copas embutidas (Fig. 1 a)) de diámetros, d, de 40, 50 y $90 \mathrm{~mm}$, respectivamente, conforme a la ecuación (1). Para ello, se ha considerado que la anchura final de las probetas estiradas, $\mathrm{h}_{1}$, es de $10,7 \mathrm{~mm}$.

$$
\alpha=\frac{2 \cdot h_{1}}{d} \cdot \frac{180}{\pi}
$$

Las probetas ensayadas se prepararon cortando tiras de chapa de acero AISI 304 en estado de recepción y con distintos estados de deformación previa, definidos en la tabla III. La anchura inicial de las probetas, $h_{o}$, se ha ido variando entre 11,5 y $15 \mathrm{~mm}$. Para poder introducir las muestras en la matriz, se mecanizó un estrangulamiento con la forma de la matriz.

Los ensayos de deformación en condiciones de tracción-compresión biaxial se realizaron utilizando como lubricante una mezcla de aceite/ $\mathrm{S}_{2} \mathrm{Mo}$ al $50 \%$ en peso. Se seleccionó este lubricante con el fin de minimizar las condiciones de rozamiento lateral producidas en el borde de la matriz.

\subsubsection{Determinación de las fuerzas de estirado y la tensión de embutición}

Los valores de la fuerza de estirado necesaria para producir una determinada deformación, $\mathrm{F}_{\mathrm{E}}$, se han determinado directamente de los registros de fuerza obtenidos, de acuerdo al procedimiento descrito en trabajos previos $^{[13]}$. Esta fuerza, es el resultado de la suma de la fuerza necesaria para realizar la deformación $\left(\mathrm{F}_{\mathrm{emb}}\right)$ y la fuerza de rozamiento $\left(\mathrm{F}_{\mathrm{R}}\right)$ del material con la matriz y el tapón en el plano de la chapa, según las ecuaciones (2) y (3), donde, m es el coeficiente de rozamiento y $\mathrm{F}_{\mathrm{N}}$, la fuerza normal aplicada sobre las matrices. 

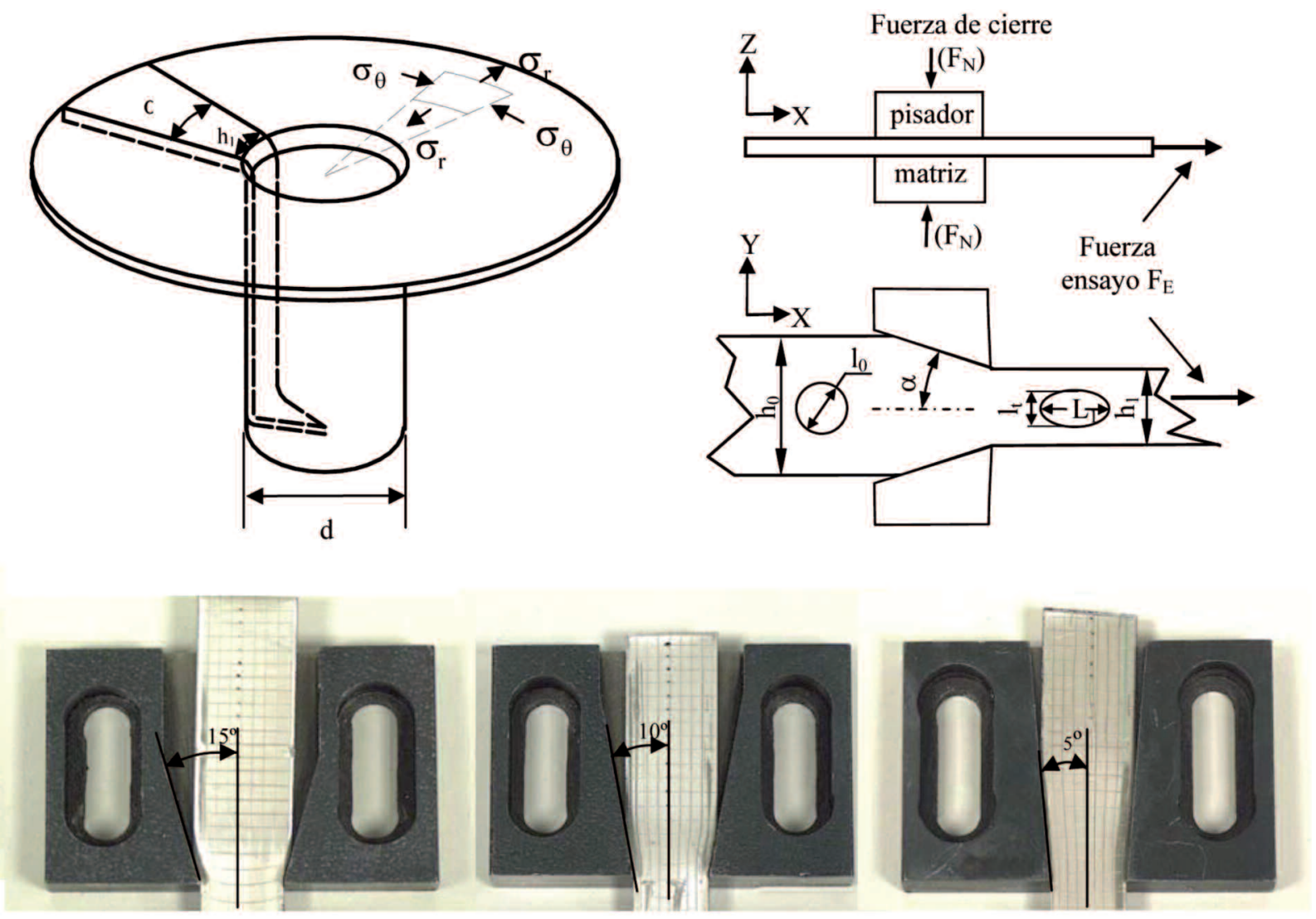

Figura 1. a) Esquema de una copa embutida; b) Detalle del ensayo de estirado T-CC; c) detalle de las matrices con las probetas embutidas.

Figure 1. a) Drawing cup scheme; b) Tensile-biaxial compression test fundamentals (T-CC); c) Test dies detail.

$$
\begin{gathered}
F_{E}=F_{e m b}+F_{R} \\
F_{R}=\mu \cdot F_{N}
\end{gathered}
$$

Los valores del coeficiente de rozamiento, en estas condiciones, se han determinado previamente mediante ensayos de rozamiento plano ${ }^{[12]}$. En este sentido se ha considerado que en presencia de aceite $/ \mathrm{S}_{2} \mathrm{Mo}$, el coeficiente de rozamiento en el rango de presiones estudiado se mantiene constante.

La determinación de la fuerza de rozamiento producida en el ensayo ha permitido determinar la fuerza necesaria para la deformación, $\mathrm{F}_{\mathrm{emb}}$. La fuerza de deformación, $\mathrm{F}_{\mathrm{emb}}$, incluye el rozamiento lateral de la chapa en la matriz.

Por analogía con el proceso de embutición, se expresa como tensión de embutición, $\sigma_{\text {emb }}$, la fuerza indicada dividida por la sección transversal de la chapa a la salida del ensayo, según la ecuación (4); $\mathrm{h}_{1}$, representa la anchura final de la muestra embutida y t, el espesor de la chapa, que se supone constante en todos los ensayos.

\subsubsection{Evaluación de las deformaciones producidas}

Para evaluar las deformaciones producidas en el material tras el ensayo, se grabaron, de forma mecánica, circunferencias de diámetro conocido, $1_{0}$ (Fig. 1b)). Las deformaciones longitudinal, $\varepsilon_{1}$, y transversal, $\varepsilon_{\mathrm{t}}$, producidas tras el ensayo pueden evaluarse conforme a las ecuaciones (5) y (6), teniendo en cuenta las dimensiones finales de las circunferencias en el sentido del estirado, $1_{1}$, y en el transvesal, $1_{t}$, respectivamente.

$$
\varepsilon_{1}=\ln \left(\frac{I_{1}}{I_{0}}\right)
$$




$$
\varepsilon_{t}=\ln \left(\frac{I_{t}}{I_{0}}\right)
$$

La deformación en el sentido del espesor de la chapa, $\varepsilon_{z}$, se obtiene a partir de $\varepsilon_{1}$ y $\varepsilon_{\mathrm{t}}$, considerando la hipótesis de volumen constante, conforme a la ecuación (7).

$$
\varepsilon_{\mathrm{z}}=\varepsilon_{\mathrm{l}}-\left|\varepsilon_{\mathrm{t}}\right|
$$

Finalmente, la deformación equivalente, $\varepsilon_{\mathrm{eq}}$, de Von Misses se obtiene conforme a la ecuación (8).

$$
\varepsilon_{\text {eq }}=\sqrt{\frac{2}{3}\left(\varepsilon_{1}^{2}+\varepsilon_{\mathrm{t}}^{2}+\varepsilon_{\mathrm{z}}^{2}\right)}
$$

La deformación en anchura, $\varepsilon_{\mathrm{h}}$, se ha determinado de acuerdo a la ecuación (9).

$$
\varepsilon_{\mathrm{h}}=\ln \left(\frac{\mathrm{h}_{1}}{\mathrm{~h}_{0}}\right)
$$

Considerando los valores de $\varepsilon_{\mathrm{h}}$ y $\varepsilon_{\mathrm{eq}}$, determinados experimentalmente mediante el procedimiento descrito y las ecuaciones (8) y (9), se ha definido una correlación lineal entre la deformación en anchura y la deformación equivalente, obteniéndose un índice de correlación de 0,95 (Ec. (10)), que permite determinar, numéricamente, la deformación equivalente a partir de la deformación en anchura determinada de forma experimental.

$$
\varepsilon_{\text {eq }}=1.136 \cdot \varepsilon_{\mathrm{h}}-0.055
$$

\subsubsection{Anisotropía del material deformado en condiciones de tracción- compresión biaxial (T-CC)}

Sobre las muestras deformadas en condiciones T-CC se ha determinado el coeficiente de anisotropía plástica, $r$, de acuerdo a la ecuación (11), en las que $b_{\mathrm{o}}$ y $\mathrm{L}_{\mathrm{o}}$ representan la anchura y longitud iniciales de la probeta; b y $L$ corresponden a los valores de anchura y longitud, tras aplicar un determinado valor de carga en el ensayo ${ }^{[16]}$.

$$
r=\frac{\ln \frac{b_{0}}{b}}{\ln \frac{L \cdot b}{L_{0} \cdot b_{0}}}
$$

Las dimensiones de las probetas ensayadas se han establecido en función de la anchura de las muestras embutidas (Fig. 2). El mecanizado de las muestras permite eliminar la zona de material expuesta al rozamiento lateral en la matriz. Esta zona presenta características microestructurales y de dureza muy diferentes a las del resto del material, que pueden afectar a los valores de anisotropía, tal y como se ha demostrado en trabajos previos ${ }^{[17]}$.

\section{RESULTADOS Y DISCUSIÓN}

\subsection{Ensayos de deformación en condiciones T-CC del material en estado de recepción. Evaluación de las condiciones de contorno del ensayo}

Los valores de la tensión de embutición del material en estado de recepción obtenidos en los ensayos realizados con las matrices de $15^{\circ}, 10^{\circ}$ y $5^{\circ}$ aparecen representados en la figura 3 . Se observa que, para una

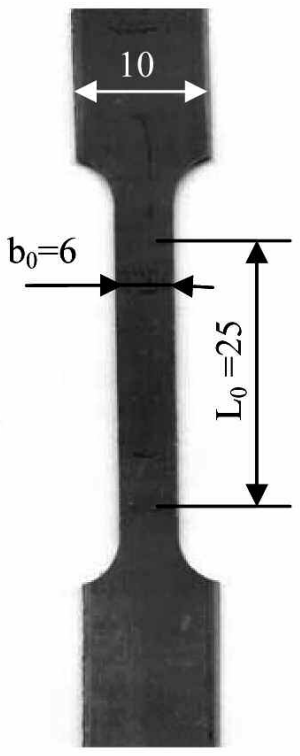

a)

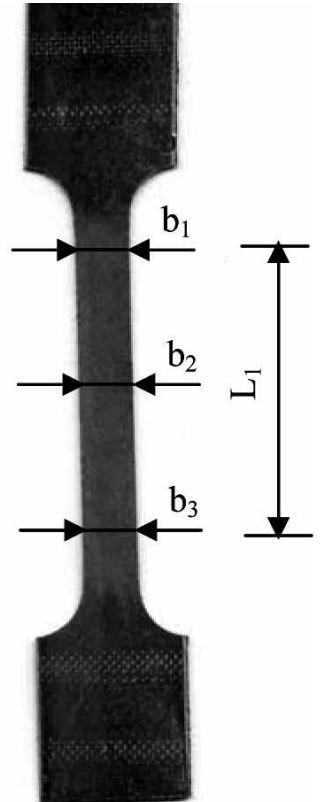

b)
Figura 2. Esquema de una probeta mecanizada: a) dimensiones iniciales; b) dimensiones después de realizar los ensayos de anisotropía.

Figure 2. Standarized tensile specimen dimensions mechanized in drawed strips: a) initial dimensions; b). Dimensions after the test of anisotropy. 


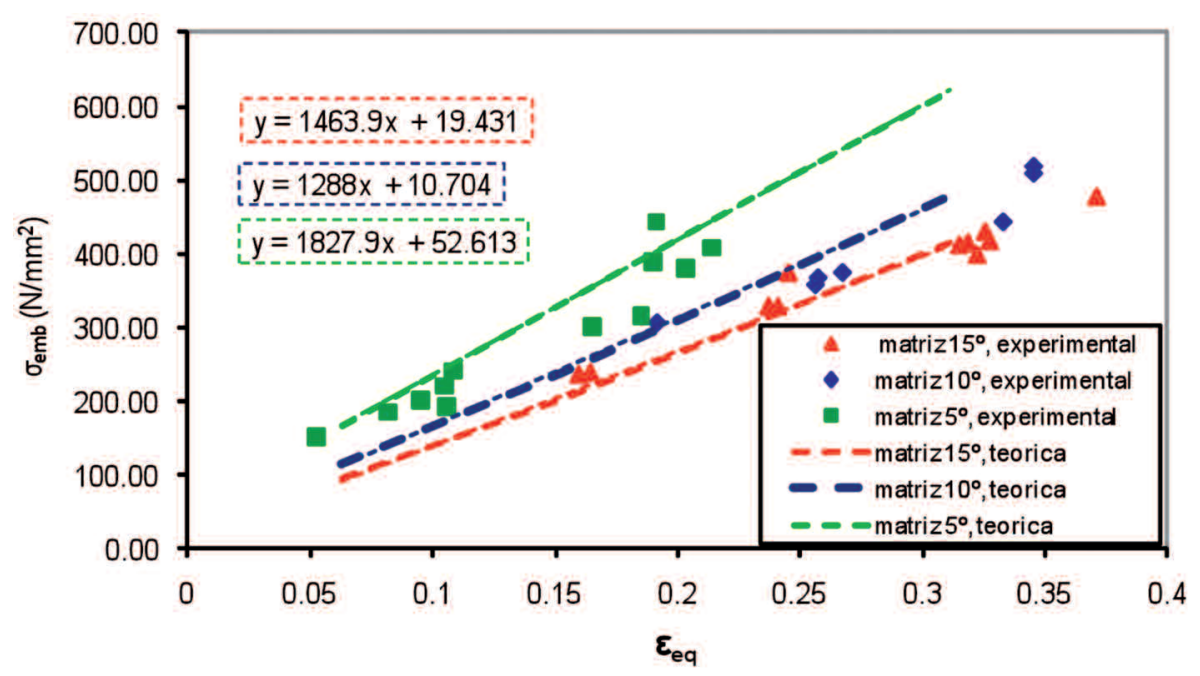

Figura 3. Tensión teórica y experimental de embutición en función de la deformación equivalente para diferentes ángulos de matriz. Muestras ensayadas en la dirección de laminación.

\section{Figure 3. Theorical and experimental drawing stress versus equivalent strain for different die angles. The assays are done for rolling direction in all cases.}

misma deformación, los valores de $\sigma_{\text {emb }}$ obtenidos en la matriz de $5^{\circ}$ son superiores a los obtenidos con las matrices de $10^{\circ}$ y $15^{\circ}$. Este hecho se debe a la influencia del diferente rozamiento lateral que experimenta el material en el ensayo, tal y como se justifica a continuación.

Los resultados experimentales obtenidos se han contrastado con los calculados de forma teórica mediante la ecuación (12), definida en el modelo de Rowe de las tensiones locales para el estirado de chapa en condiciones de deformación plana ${ }^{[18]}$. Para ello, se ha extrapolado el modelo mencionado cambiando el plano general de la chapa, dado que la condición de invariabilidad dimensional se produce en el espesor, en el caso de la embutición. Esto es, se exporta el modelo de comportamiento de deformación plana a uno típico de pure shear, invirtiendo la dimensión del espesor en el proceso de estirado con el plano principal de la chapa en la embutición. En la ecuación (11), se aúnan el coeficiente de fricción lateral, $\mu_{\mathrm{L}}, \mathrm{y}$ el ángulo de la matriz en un único término, $\mathrm{B}$, conforme a la ecuación (13).

$$
\begin{gathered}
\sigma_{\text {emb }}=\left(\frac{1+B}{B}\left(1-\frac{h_{1}}{h_{0}}\right)^{B}\right) \cdot 1.115 \cdot \sigma_{\text {fluencia }} \\
B=\mu_{L} \cdot \operatorname{cotg}(\alpha / 2)
\end{gathered}
$$

En la figura 3, se representan los valores de la tensión de deformación para cada uno de los ángulos en función de la deformación practicada, observándose que los valores teóricos concuerdan, en gran medida, con los experimentales para los tres ángulos estudiados. La tendencia creciente del valor de la tensión para ángulos menores debido a la fuerza de rozamiento lateral, se demuestra en ambos métodos.

Para evaluar de forma analítica la fuerza de rozamiento lateral producida en el ensayo T-CC se ha establecido un balance de las diferentes fuerzas que actúan en el eje X o eje de estirado (Fig. 4), conforme a la ecuación (14), donde, $\mathrm{F}_{\mathrm{N \alpha}}$ y $\mathrm{F}_{\mathrm{RL \alpha}}$ representan la fuerza normal ejercida sobre el borde de la matriz y

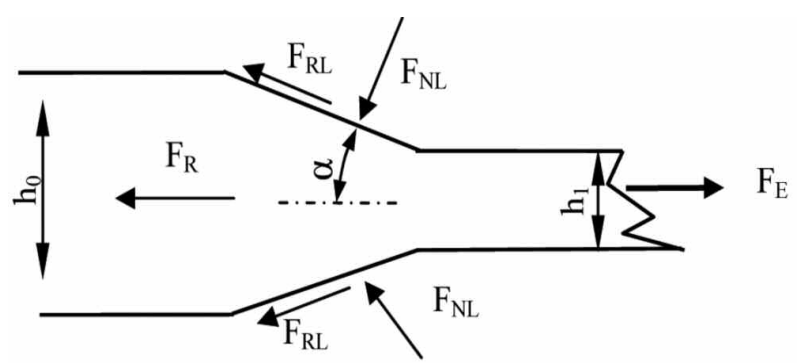

Figura 4. Esquema de las fuerzas que intervienen en el ensayo de deformación T-CC.

Figure 4. Detail of forces that take place wedge shape test. 
la fuerza de rozamiento correspondiente en función del ángulo, $\alpha$, considerado para la matriz.

$$
\mathrm{F}_{\mathrm{emb}}=2 \cdot \mathrm{F}_{\mathrm{NL \alpha}} \cdot \operatorname{sen} \alpha+2 \cdot \mathrm{F}_{\mathrm{RL} \alpha} \cdot \cos \alpha
$$

Teniendo en cuenta la relación existente entre la fuerza de rozamiento lateral y la fuerza normal basada en el coeficiente de rozamiento lateral existente, $\mu$, se puede finalmente simplificar la expresión (14) como función, únicamente, de $\mathrm{F}_{\mathrm{N \alpha}}$, tal y como se indica en la ecuación (15) ya que, $\mu$ ha sido determinado previamente tal y como se ha expuesto con anterioridad.

$$
\mathrm{F}_{\mathrm{emb}}=2 \cdot \mathrm{F}_{\mathrm{N} \alpha} \cdot \operatorname{sen} \alpha+2 \cdot \mu \cdot \mathrm{F}_{\mathrm{N \alpha}} \cdot \cos \alpha
$$

A partir de la expresión (15) se obtienen los valores de $\mathrm{F}_{\mathrm{N}}$. para los ensayos realizados y, a partir de éstos, los valores de $F_{R L}$. Los valores obtenidos se han correlacionado con la deformación equivalente (Fig. 5), obteniéndose una función lineal para cada una de las matrices utilizadas

Los resultados demuestran que el ángulo de la matriz influye de forma muy significativa sobre la fuerza de rozamiento lateral. Los valores de ésta son comparables a los obtenidos de forma teórica, previamente, por los autores mediante el método de las líneas de deslizamiento ${ }^{[8]}$, demostrándose que para ángulos de conicidad inferiores a $15^{\circ}$ la fuerza de rozamiento lateral es muy elevada e influye de forma muy significativa sobre los valores de la tensión de estirado.

A pesar de lo indicado, el ensayo T-CC reproduce bien los estados de deformación en condiciones

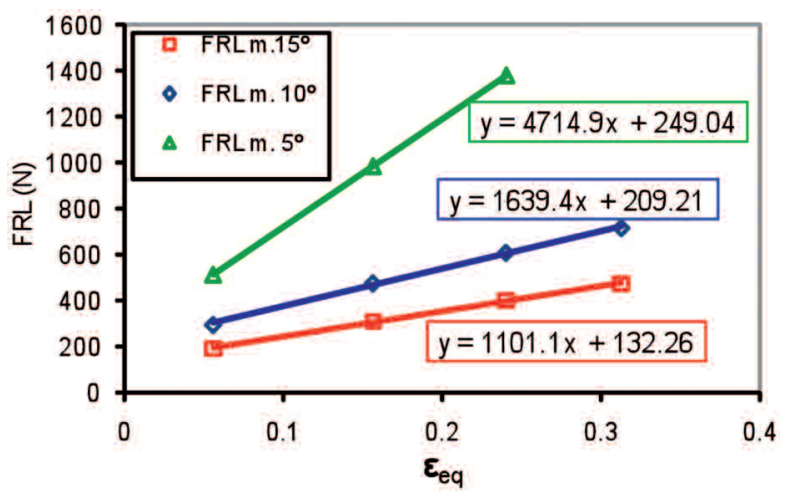

Figura 5. Fuerza de rozamiento lateral en función de la deformación equivalente para diferentes ángulos de matriz. Muestras ensayadas en la dirección de laminación.

Figure 5. Lateral friction force versus equivalent strain for different die angles. The assays are made for rolling direction. de embutición, por lo que un ángulo de matriz grande minimiza los efectos de contorno de aquél.

\subsubsection{Evaluación de la deformación tras el ensayo}

Para evaluar las deformaciones producidas en el ensayo de T-CC, por analogía con el coeficiente de anisotropía normal, se ha utilizado un coeficiente de deformación, $\delta$, que establece la relación entre la deformación transversal, $\varepsilon_{t}$ y la deformación en espesor, $\varepsilon_{7}$, definido previamente para acero DC-0 $5^{[13]}$. Considerando la hipótesis de invariabilidad de volumen, se ha expresado el coeficiente $\delta$ en función de las deformaciones longitudinales y transversales experimentadas por el material en el plano de la chapa, según la ecuación (16).

$$
\delta=\frac{\ln \left(\frac{I_{0}}{I_{t}}\right)}{\ln \left(\frac{I_{t} \cdot I_{1}}{I_{0}^{2}}\right)}
$$

Dado que la variación de espesor es muy pequeña, el coeficiente $\delta$ alcanza valores muy elevados, por lo que se ha considerado la función inversa de este valor para el análisis de resultados.

En la figura 6 se representan los valores obtenidos para $1 / \delta$ frente a la deformación equivalente producida sobre muestras deformadas en condiciones de TCC. Se han considerado las deformaciones producidas sobre muestras con diferentes grados de acritud inicial. Como se puede observar, el valor de $1 / \delta$ es función de la deformación en el ensayo, no existiendo influencia del estado de acritud inicial del material. El coeficiente $1 / \delta$ disminuye con la deformación equivalente, de acuerdo a una función exponencial decreciente con tendencia a cero. Este comportamiento indica que para pequeñas deformaciones equivalentes, inferiores a 0,15 , la deformación debida a la tensión longitudinal de tracción por las condiciones de contorno establecidas en el ensayo es superior a la transversal de compresión y, como consecuencia de ello, existe adelgazamiento del material. Sin embargo, para deformaciones superiores este factor se aproxima a cero, lo que demuestra que la variación de espesor es muy pequeña. Por tanto, puede considerarse que para deformaciones superiores a 0,15 los ensayos realizados se han desarrollado en condiciones de pure shear, típicas de embutición profunda. Estos resultados corroboran los obtenidos para acero DC-05 [13]. 


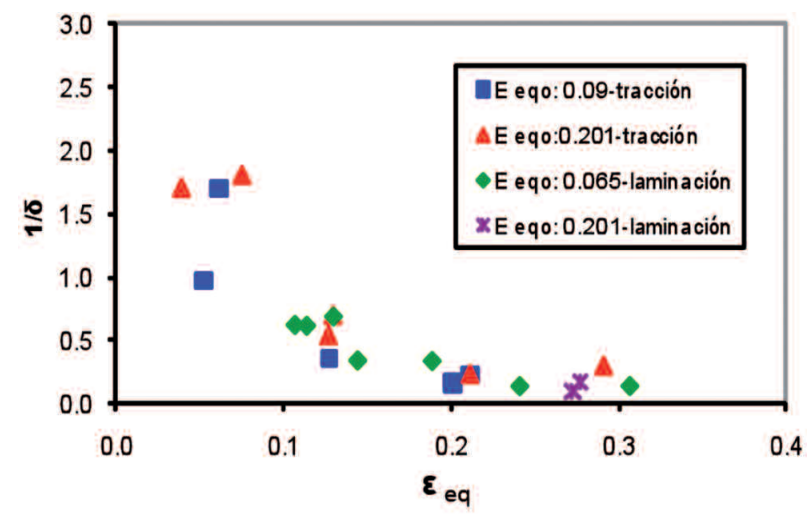

Figura 6. Variación del factor $1 / \delta$ con la deformación equivalente para diferentes grados de acritud inicial.

Figure $6.1 / \delta$ values as a equivalent strain function, $e_{\text {eq }}$, for different initial strains.

\subsection{Ensayos de deformación en condiciones T-CC del material sometido a diferentes grados de acritud}

Para comprobar el efecto de la acritud del material de partida sobre la tensión de embutición, se han ensayado varias series de probetas con diferentes grados de acritud inicial. La deformación equivalente de Von Mises, $\varepsilon_{\text {eqo }}$, de estas muestras, ha sido de 0,090 y 0,205 producida mediante tracción y de 0,065 y 0,180 mediante laminación. También, se ha realizado una serie de muestras en estado de recepción, considerándolas como sin acritud

En la figura 7 aparecen representados los valores de la tensión de embutición en función de la deformación para los distintos grados de acritud inicial. En ella se observa, que para una misma deformación equivalente producida en el ensayo, la tensión de embutición aumenta con la acritud inicial. También, se observa que las tensiones de embutición necesarias para deformar las muestras previamente sometidas a esfuerzos de tracción son muy similares a las obtenidas para probetas laminadas con una deformación del mismo orden, por lo que se demuestra que para el rango de deformaciones estudiadas, el efecto de la acritud por laminación y tracción uniaxial sobre la tensión de embutición, es del mismo orden.

Los datos de tensión de embutición obtenidos para cada grado de acritud se han ajustado a una función potencial, similar a la de Hollomon, en las que se observa que el exponente o índice de endurecimiento disminuye a medida que aumenta el grado de acritud inicial. En la figura 8, se ha representado el coeficiente de endurecimiento frente a la deformación equivalente del material producida mediante laminación y tracción. Los resultados obtenidos demuestran que el proceso de deformación previo del material, en el rango de deformaciones estudiado, no influye sobre el endurecimiento del material producido en el proceso de deformación en condiciones T-CC.

Para establecer una única función que relacione la tensión de embutición con el grado de deformación producido en condiciones de tracción-compresión biaxial, $\varepsilon_{\text {eq }}$, y del grado de acritud inicial del

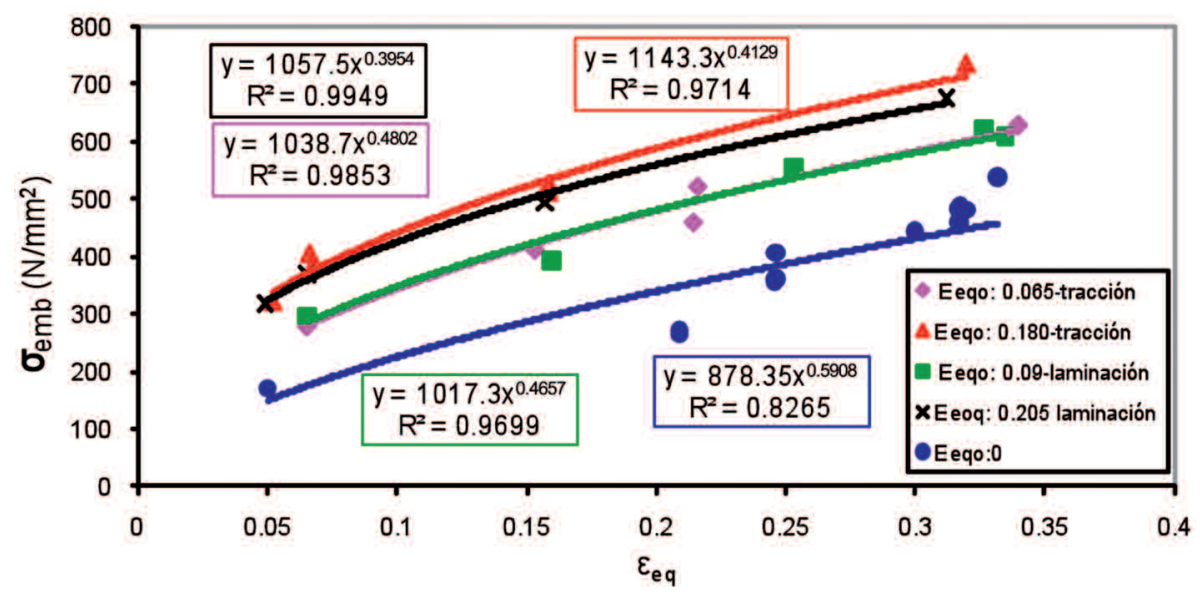

Figura 7. Tensión de embutición frente a la deformación equivalente en T-CC para diferentes grados de acritud inicial producidos mediante laminación y tracción uniaxial.

Figure 7. Drawing stress versus equivalent strain for different initial strain grades by cold rolling and tensile force. 


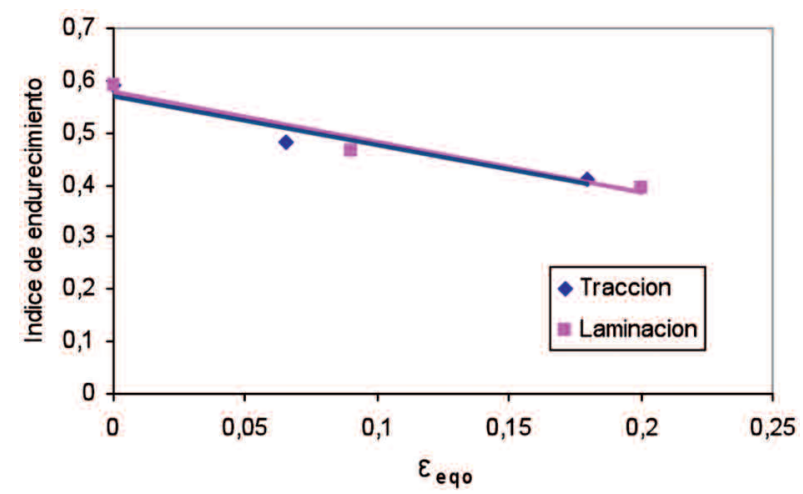

Figura 8. Variación del coeficiente de endurecimiento con la acritud previa del material.

Figure 8. Strengthening coefficients changing with previous deformation of the material.

material, $\varepsilon_{\text {eqo }}$, se ha determinado un ajuste lineal de acuerdo a la ecuación (17), en la que se establece el índice de correlación obtenido, $\mathrm{R}^{2}$.

$$
\sigma_{\text {emb }}=115+1435 \cdot \varepsilon_{\text {eqo }}+1026 \cdot \varepsilon_{\text {eq }} R^{2}: 0,83
$$

El análisis de la función permite establecer que el efecto de la acritud inicial es predominante en relación a la influencia de la deformación producida posteriormente en el proceso T-CC, independientemente del estado tensional asociado.

\subsection{Determinación de la anisotropía del material con la evolución del proceso de deformación en condiciones de compresión biaxial}

\subsubsection{Influencia del ángulo de la matriz}

Para determinar la influencia del ángulo de la matriz sobre las propiedades plásticas del material durante el proceso de embutición se determinó la anisotropía del material, r, en función de la deformación equivalente. En la figura 9 aparecen representados los valores de r, para cada uno de los ángulos estudiados.

En esta gráfica se constata que el ángulo de la matriz no influye sobre las propiedades del material embutido, lo que es consistente con los resultados indicados con anterioridad, relativos al efecto de contorno lateral de la matriz. El ángulo de la matriz no influye sobre el estado de deformación aplicado sobre el material, especialmente, a partir de una deformación mínima.

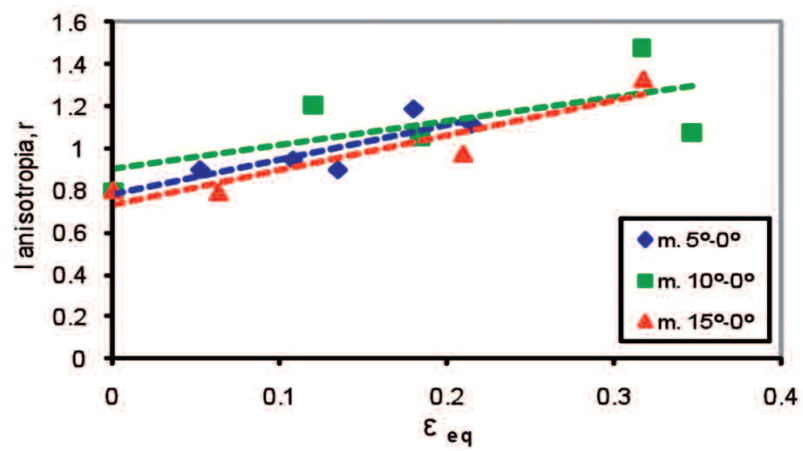

Figura 9. Variación del índice de anisotropía, r, en la dirección de laminación $\left(0^{\circ}\right)$ con la deformación equivalente. Influencia del ángulo de la matriz de deformación.

Figure 9. Anisotropy coefficient values, $r$, versus equivalent strain for the in rolling direction. Deformation die angle influence on $r$.

Se observa que el índice de anisotropía aumenta con el grado de deformación, lo que demuestra la predicción de ciertos autores ${ }^{[19]}$ que justifican sus resultados basándose en la hipótesis de la variación del coeficiente de anisotropía con la deformación ${ }^{[2]}$.

En la figura 10 se ha representado el cociente $\mathrm{r} / 1+\mathrm{r}$, equivalente a la relación entre las tensiones circunferencial y radial, $\sigma_{\theta} / \sigma_{\mathrm{r}}$ en la embutición de copas $^{[1]}$, frente a la deformación. Se observa que los resultados obtenidos, independientes del ángulo de la matriz, son comparables a los obtenidos por algunos autores ${ }^{[2]}$ que demuestran, analíticamente, que la relación $\sigma_{\theta} / \sigma_{\mathrm{r}}$ no depende del tamaño de la copa, sino de la relación de embutición (D/d).

\subsubsection{Influencia de la dirección de laminación sobre el coeficiente de anisotropía}

Con objeto de determinar la influencia de la dirección de laminación sobre los valores de anisotropía del material conformado, se han realizado ensayos de anisotropía sobre muestras embutidas en la dirección de $0^{\circ}, 45^{\circ}$ y $90^{\circ}$ con respecto a la dirección de laminación. Los resultados obtenidos aparecen representados en la figura 11. Tal y como se ha indicado previamente, se observa que las muestras ensayadas a $0^{\circ}$, presentan una tendencia creciente del coeficiente de anisotropía con el grado de deformación. Por el contrario para 45 y $90^{\circ}$, no se observa una tendencia claramente definida. También se representa el valor del coeficiente de anisotropía normal promediado para las diferentes direcciones de laminación conforme a la ecuación (18). 


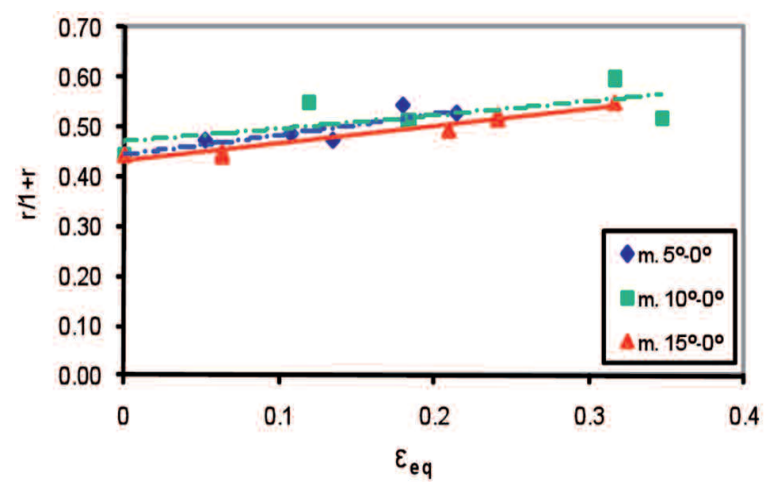

Figura 10. Variación de la función $(r / 1+r)$ con la deformación equivalente en el material en la dirección de laminación $\left(0^{\circ}\right)$. Influencia del ángulo de la matriz de deformación.

Figure 10. (r/1+r) function value versus equivalent strain in the rolling direction and the die angle influence on that.

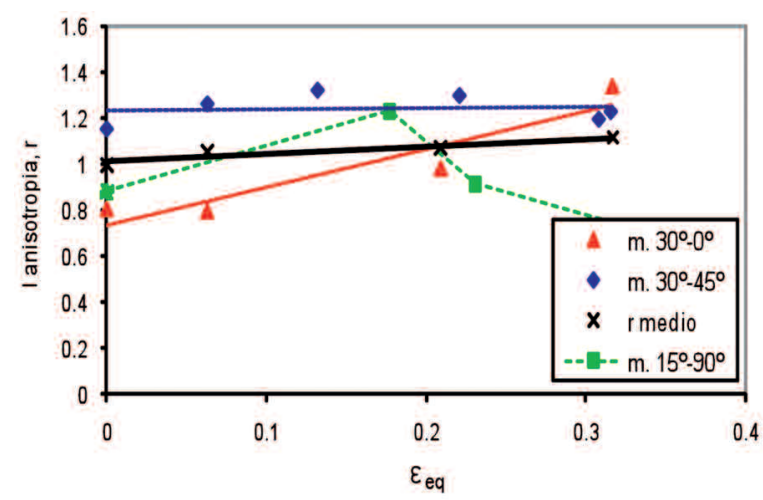

Figura 11. Valores del índice de anisotropía en función de la deformación equivalente de muestras deformadas en la matriz de $15^{\circ}$. Influencia de la dirección de laminación.

Figure 11. Anisotropy coefficient value as a function of the equivalent strain in with a die angle of 15. Influence of the rolling direction.

$$
r=\frac{r_{0}+2 r_{45}+r_{90}}{4}
$$

Estos resultados se han comparado con los valores de anisotropía del material deformado mediante laminación y tracción uniaxial (Figs. 12 y 13), constatándose que las muestras deformadas en la dirección de $0^{\circ}$ presentan la misma tendencia, es decir, se produce un ligero incremento de los valores de anisotropía con la deformación. Esta tendencia es similar a la obtenida por Kawai en acero galvanizado ${ }^{[19]}$.
El coeficiente de anisotropía normal obtenido sobre las distintas muestras deformadas mediante los distintos mecanismos de deformación es, prácticamente, coincidente para los tres procesos de acritud experimentados, T-CC, laminación y tracción uniaxial.

\section{CONCLUSIONES}

- En el presente trabajo se ha experimentado el comportamiento del acero inoxidable AISI 304 DDQ mediante un proceso de deformación en condiciones de tracción-compresión biaxial. Se ha

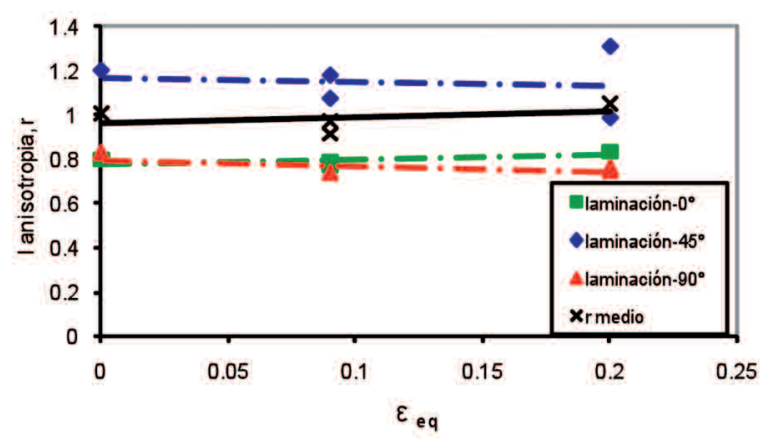

Figura 12. Valores del coeficiente de anisotropía frente a la deformación equivalente de muestras laminadas. Influencia de la dirección de laminación.

Figure 12. Anisotropy coefficient value versus equivalent strain in rolling samples. Influence of the rolling direction.

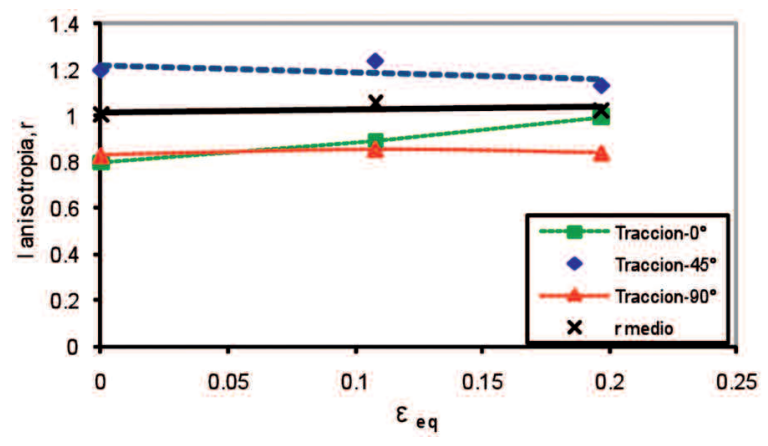

Figura 13. Valores del coeficiente de anisotropía frente a la deformación equivalente de muestras deformadas mediante tracción uniaxial. Influencia de la dirección de laminación

Figure 13. Anisotropy coefficient value versus equivalent strain for deformed samples by uniaxial tensile force. Influence of the rolling direction. 
demostrado que el procedimiento de ensayo reproduce perfectamente las condiciones de deformación plana producidas en la embutición para deformaciones equivalentes superiores a 0,15. Para deformaciones inferiores, se produce un ligero adelgazamiento del material como consecuencia de las condiciones de contorno que introduce el ensayo. No obstante, aún para estas bajas deformaciones, el ensayo es representativo de las condiciones de deformación que experimentan los procesos de embutición de chapa.

- Se han obtenido los coeficientes de anisotropía normal, $r$, y de endurecimiento, $\mathrm{n}$, del acero para distintas deformaciones obtenido bajo condiciones multiaxiales típicas del proceso de embutición estableciendo la variación de dichos índices. Se ha demostrado que existe un ligero aumento de $r$, con la deformación. Los valores de anisotropía del material con distintos grados de deformación equivalente confirman que la relación $\sigma_{\mathrm{q}} / \sigma_{\mathrm{r}}$ es independiente del ángulo de la matriz.

- Se ha establecido la influencia del estado de acritud inicial del material sobre el proceso de deformación en condiciones de tracción-compresión biaxial típicas del proceso de embutición. Los resultados indican que la deformación inicial en el material ejerce un efecto predominante respecto de las deformaciones propias del proceso T-CC. No existe influencia del modo de deformación previo practicado, tracción uniaxial y laminación.

\section{Nomenclatura}

$\alpha \quad$ Ángulo de la matriz cónica

$\mu \quad$ Coeficiente de rozamiento

$\delta \quad$ Coeficiente de adelgazamiento

$\sigma_{\theta} \quad$ Tensión circunferencial

$\sigma_{r} \quad$ Tensión radial

T-CC Tracción-compresión biaxial

$\varepsilon_{\text {eq }} \quad$ Deformación equivalente de Von Misses en TCC

$\varepsilon_{\text {eqo }} \quad$ Deformación equivalente de Von Misses inicial

$\varepsilon_{\mathrm{h}} \quad$ Deformación en anchura

$\varepsilon_{1} \quad$ Deformación longitudinal

$\varepsilon_{t} \quad$ Deformación transversal

$\varepsilon_{z} \quad$ Deformación en espesor

$\mu_{\mathrm{L}} \quad$ Coeficiente de rozamiento lateral

d Diámetro de copa

$\mathrm{F}_{\mathrm{E}} \quad$ Fuerza de estirado

$\mathrm{F}_{\mathrm{emb}} \quad$ Fuerza de embutición

$\mathrm{F}_{\mathrm{N}} \quad$ Fuerza normal de cierre en las matrices.

$\mathrm{F}_{\mathrm{NL}} \quad$ Fuerza normal lateral

$\mathrm{F}_{\mathrm{R}} \quad$ Fuerza de rozamiento

$\mathrm{F}_{\mathrm{RL}} \quad$ Fuerza de rozamiento lateral $\mathrm{h}_{1} \quad$ Anchura final de la probeta tras el ensayo

$h_{0} \quad$ Anchura inicial de la probeta de ensayo

$l_{1} \quad$ Dimensión longitudinal de las marcas tras el ensayo

1。 Diámetro inicial de marcas

$l_{t} \quad$ Dimensión transversal de las marcas tras el ensayo

n Coeficiente de endurecimiento

$\mathrm{L}_{\mathrm{o}} \quad$ Longitud calibrada de la probeta

$\mathrm{L}_{1} \quad$ Longitud de la probeta tras la deformación

r Índice de anisotropía

t Espesor de la chapa

\section{REFERENCIAS}

[1] D. C. Chiang y S. Kobayashi, J. Eng. Ind. (1966) 443-448.

[2] D. M. Woo, J. Mech. Eng. Sci. 2 (1964) 116-131.

[3] E. Gao, W. Li, H. Kou, H. Changa, J. Li y L. Zhou, Trans. Nonferrous Met. Soc. China 19 (2009) 433-437.

[4] C. García, D. Celentano, F. Flores, J.P. Ponthot y O. Oliva. J. Mater. Proc. Technol. 172 (2006) 461-471.

[5] S. Holmberg, B. Enquist y P. Thilderkvist, J. Mater. Process. Tech. 147 (2004) 72-83.

[6] J.L. Geoffroy, J. Goncalves y X. Lemoine, IDDRG 2007 Conf., Györ, Hungría, pp.177-180.

[7] L. Kessler, H. Richter, K. Roll y A. Faust, IDDRG 2007 Conf., Györ, Hungría, pp. 101-108.

[8] V. Miguel, J. Benet, J. Coello, A. Calatayud y A. Martínez, Mater. Proc. Design. Model., Simul. Appl. (2007) pp. 823-828.

[9] ASM Handbook. Vol. 14B (2005) pp. 673-696.

[10] S. Rajagopal, J. Eng. Ind. 103 (1981) 197-202.

[11] L. Gunnarson, N. Asnafi y E. Schedin, J. Mater. Process. Tech. 73 (1998) 89-96.

[12] T. Yagami, K. Manabe y Y. Yamauchi, J. Mater. Process. Tech. (2007) 187-191.

[13] V. Miguel, C. Ferrer y A. Calatayud, Rev. Metal. Madrid 43 (2007) 146-156.

[14] V. Miguel, J. Coello, A. Calatayud, M.C. Manjabacas, A. Martínez y C. Ferrer. J. Mater. Process. Tech. 209 (2009) 1.588-1.596.

[15] J. Coello, V. Miguel, A. Calatayud, C. Ferrer y A. Martínez, Rev. Metal. Madrid 44 (2008) 503-512.

[16] UNE EN ISO 36404:1988 EX.

[17] J. Coello, V. Miguel, C. Ferrer, A. Calatayud y A. Martínez, Rev. Metal. Madrid 45 (2009) 351 364.

[18] G. W. Rowe, Conformado de los metales, Ed. Hurmo, Bilbao, 1972, pp. 86-95.

[19] N. Kawai, N. Hayashi y S. Matsui, Trans. ASME 110 (1988) 376-383. 